\title{
On Narrowband I nterference Suppression in OFDM-based Systems with CDMA and Weighted-type Fractional Fourier Transform Domain Preprocessing
}

\author{
Yuan Liang, Xinyu Da and Shu Wang \\ School of Information and Navigation Institute, Air Force Engineering University \\ Xi'an, Shaanxi 710077- CHINA \\ [e-mail: 88048406@qq.com, daxinyu@vip.sina.com,xss_wang@163.com] \\ *Corresponding author: Yuan Liang
}

Received September 24, 2016; revised December 15, 2016; revised June 27, 2017; accepted July 20, 2017; published November 30, 2017

\begin{abstract}
In this paper, we propose a new scheme to suppress the narrowband interference (NBI) in OFDM-based systems. The scheme utilizes code division multiple access (CDMA) and weighted-type fractional Fourier transform (WFRFT) domain preprocessing technologies. Through setting the WFRFT order, the scheme can switch into a single carrier (SC) or a multi-carrier (MC) frequency division multiple access block transmission system. The residual NBI can be eliminated to the maximum extent when the WFRFT order is selected properly. Final simulation results show that the proposed system can outperform MC and SC with CDMA and frequency domain preprocessing in terms of the narrowband interference suppression.
\end{abstract}

Keywords: narrowband interference (NBI), weighted-type fractional Fourier transform (WFRFT), transform domain preprocessing, code division multiple access (CDMA), orthogonal frequency division multiplexing (OFDM) 


\section{Introduction}

$\mathbf{N}_{\text {owadays, Long Term Evolution (LTE) system has already been put into commercial }}$ operation. LTE takes the OFDM technology as its downlink and single carrier frequency division multiple access (SC-FDMA) as its uplink. SC-FDMA has lower peak-to-average power ratio (PAPR) and higher power efficiency at the mercy of lower spectrum usage, while OFDM has higher spectrum usage at the mercy of higher PAPR [1,2]. In order to acquire the merits of both single carrier(SC) and Multi-carrier (MC), Many efforts have been made to converge the SC and MC system [3]. Meilin firstly introduced a hybrid carrier (HC) [4,5] communication system based on weighted-type fractional Fourier transform (WFRFT), which can be adjusted flexibly between SC and MC system. It has been confirmed that WFRFT-based system can make the signal bit energy distributed in frequency-time plane. Compared with the SC and MC system, HC communication system can help enhance the system performance to a certain extent, especially over doubly selective channels [6]. A great deal of research has been done on the HC operation and application. In [7,8], it has been verified that HC communication system can get a better bit error rate (BER) performance in the single frequency interference and additive white Gaussian noise (AWGN) channel with code division multiple access (CDMA) precoding. HC communication system can enhance the system performance when the WFRFT order $\alpha$ is properly selected during the same communication channel state $[6,9,10]$. It has also been proved that HC communication system still perform well in the communication channels with severe inter-symbol interference (ISI) and inter-carrier interference (ICI) [11]. Therefore, HC communication system will be a potential technology in the future communication system.

Since most of the existing broadband block-based transmission systems adopt OFDM-based structure, the HC communication system also adopts OFDM-based structure in transmission and reception units in order to be compatible with the current communication system. However the OFDM-based communication system are easier to suffer a severe disadvantage in being contaminated by the NBI, which has long attracted many researchers' attention $[12,13]$. And there are many kinds of methods to suppress NBI. According to the different ways to suppress the interference, these suppression methods can fall into three categories: elimination [14,15], timelfrequency domain filtering [16,17], and frequency domain preprocessing $[18,19,20]$. The basic principle of elimination method is to detect the NBI's characteristic parameters and reconstruct the NBI based on these parameters. By eliminating the reconstructed NBI from the final receiving signal, the transmitted signal will be demodulated to the greatest extent. However the elimination method is highly dependent on the parameters' accuracy and has high computational complexity [14]. As for filtering, by designing high performance filters to filter the NBI in time or frequency domain, the timelfrequency domain filtering method can enhance the anti-interference property. While in the same time, it will incur the performance reduction due to the useful signal's energy loss in the filter's stop bands and the high performance filters are also difficult to realize. Frequency domain preprocessing often does well in anti-NBI because of its capability to spread the interference into the total frequency band [21].

Based on the theories and methods above, this paper proposes the CMDA-WFrDCS (weighted-type fractional Fourier transform domain communication system) communication system, combining the CDMA with WFrDCS preprocessing technologies. The original signal is spread by a m-sequence firstly and then modulated by WFRFT. And last the NBI is 
eliminated to the most extent in the frequency domain. In order to verify the performance of CMDA-WFrDCS system designed in this paper, the formula of the WFRFT optimal order is deduced, and the capacity to anti-NBI is also analyzed.

The rest sections of this paper are organized as follows. In section 2, the overview of the novel CMDA-WFrDCS structure is introduced. In section 3, the BER performance is analyzed and the expression for the order optimization is also derived. In section 4, simulations are conducted to validate the theory and derivation, and the results are also displayed. Conclusions and future work are drawn in the last section.

\section{Architecture of CMDA-WFrDCS System}

The architecture of CMDA-WFrDCS system is depicted in Fig. 1. For clarity, we will use Tx and $\mathrm{Rx}$ to denote transmitting and receiving parts respectively. At Tx, firstly, the original baseband modulated data $s$ is spread by the pseudo sequence $\boldsymbol{d}$. Then through the $L$-points $-\alpha$ th WFRFT, the signal is distributed in both frequency and time domain. The $N$-points inverse discrete Fourier transform (IDFT), cyclic prefix (CP) and digital to analog (D/A) processes are quite the same as the conventional OFDM Tx and Rx module. The CP module can be added or not according to the channel state. When the channel is only influenced by the AWGN, the CP module can be omitted. When the channel includes the effects of both multipath fading and AWGN, the CP module is imperative to realize the channel estimation, and the frequency domain equalization (FDE) is also needed to realize the equalization according to the channel estimation. The receiver has its counterpart modules with the transmitter. More details about the CMDA-WFrDCS architecture will be stated in the following three submodules: WFRFT module, OFDM-based transform domain module and Parameter Pool module.

Compared with the architecture of HC CDMA system in [7], the proposed architecture of this paper has the frequency domain prepocessing, which can help to avoid most of the strong interferences. Moreover, unlike the architecture in [10], this paper adopts the PN sequence to spread the interference more widely in time-frequency domain.

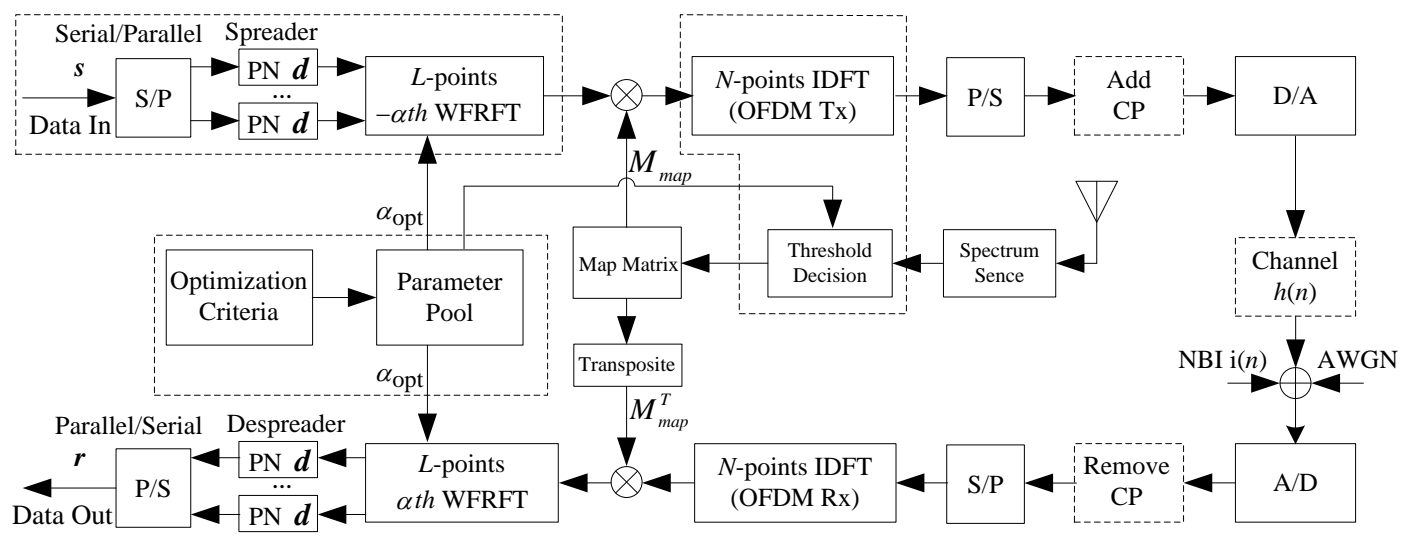

Fig. 1. Architecture of the CMDA-WFrDCS system

\subsection{WFRFT Preprocessing}

As shown in Fig. 1, through serial to parallel (S/P) conversion, a serial consecutive data are divided into a block, whose length is $L$. The $L$-length block can be denoted as the column vector $\boldsymbol{s}=\left[s_{0}, s_{1}, s_{2} \cdots, s_{L-1}\right]^{T}$. then the original modulated signal $\boldsymbol{s}$ is spread by the pseudo 
sequence $\boldsymbol{d}$, where $\boldsymbol{d}=\left[d_{0}, d_{1}, d_{2}, \cdots, d_{M-1}\right]$. After $\mathrm{S} / \mathrm{P}$ conversion and spreading module, the transmitted signal $\boldsymbol{D}$, can be expressed as

$$
\boldsymbol{D}=\left[\begin{array}{ccccc}
s_{0} d_{0} & s_{0} d_{1} & s_{0} d_{2} & \cdots & s_{0} d_{M-1} \\
s_{1} d_{0} & s_{1} d_{1} & s_{1} d_{2} & \cdots & s_{1} d_{M-1} \\
\vdots & \vdots & \vdots & \vdots & \vdots \\
s_{L-1} d_{0} & s_{L-1} d_{1} & s_{L-1} d_{2} & \cdots & s_{L-1} d_{M-1}
\end{array}\right]
$$

Then through WFRFT modulation, the transmitted signal $\boldsymbol{D}$ can be transformed into $\boldsymbol{X}$, which can be given by

$$
\boldsymbol{X}=\boldsymbol{W}_{-\alpha} \boldsymbol{D}
$$

$\boldsymbol{W}_{-\alpha}$ is the WFRFT matrix with the order , $-\alpha$, which can be expressed as follows [22]

$$
\boldsymbol{W}_{-\alpha}=w_{0}(-\alpha) \boldsymbol{F}^{0}+w_{1}(-\alpha) \boldsymbol{F}^{1}+w_{2}(-\alpha) \boldsymbol{F}^{2}+w_{3}(-\alpha) \boldsymbol{F}^{3}
$$

Where, $\boldsymbol{F}$ is the $L$-point normalized discrete Fourier transform (DFT) matrix, the expression for it is

$$
F_{m, n}=\frac{1}{\sqrt{L}} \exp (-j 2 \pi m n / L), m, n=0,1, \cdots, L-1
$$

$w_{l}(-\alpha)$ is the weighting coefficients, the expression for it is [23]

$$
w_{l}(-\alpha)=\frac{1}{4} \sum_{n=0}^{3} \exp \left(\frac{j \pi n(-\alpha-l)}{2}\right), l=0,1,2,3
$$

The framework for WFRFT module is depicted in Fig. 2. The serial signal is firstly converted from serial to parallel, which contains four branches.

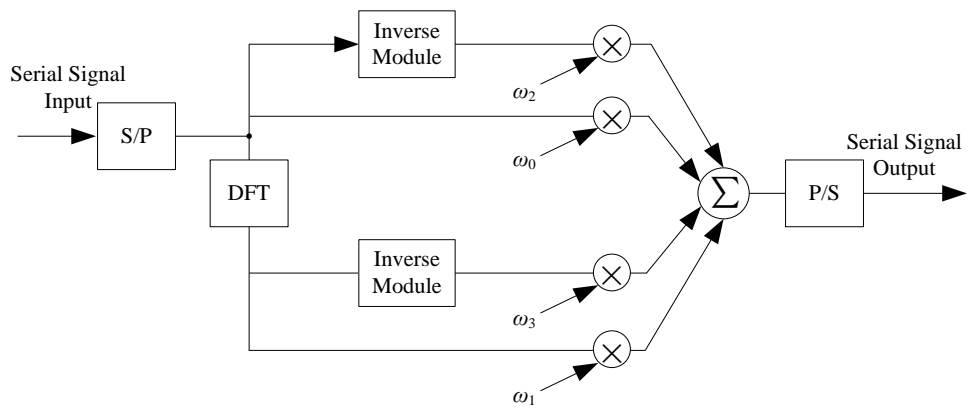

Fig. 2. Framework of WFRFT 
After the S/P conversion, through DFT and Inverse modules, the four branches are weighted with the corresponding coefficients $\omega_{2}, \omega_{0}, \omega_{3}$ and $\omega_{1}$ respectively. Then a summation of the different four branches is executed. Finally through P/S conversion, the WFRFT-modulated serial signal is output.

Then we can rewrite the expression of $\boldsymbol{W}_{-\alpha}$, whose $(m, n)$ th element is shown as follows

$$
\begin{gathered}
W_{-\alpha}(m, n)=w_{0}(-\alpha) \delta(m-n)+w_{1}(-\alpha)(1 / \sqrt{L}) \exp (-j 2 \pi m n / L) \\
+w_{2}(-\alpha) \delta\left(\langle m+n\rangle_{L}\right)+w_{3}(-\alpha)(1 / \sqrt{L}) \exp (j 2 \pi m n / L)
\end{gathered}
$$

\subsection{OFDM-based Structure}

Compared with the conventional OFDM-based structure, instead of adopting a fixed spectrum allocation strategy, the OFDM transform domain module in this paper zeros those frequencies where strong interferences exist, which can help to avoid the severe interference and improve the BER performance. According to the expression in Eq.(2), $\boldsymbol{X}$ is a $L$-by- $M$ matrix. For simplicity's sake, $\boldsymbol{X}$ is processed column by column in the OFDM-based module. And $\boldsymbol{x}^{(i)}$ denotes the ith column of $\boldsymbol{X}, \boldsymbol{x}^{(i)}=\left[\boldsymbol{X}_{0, i}, \boldsymbol{X}_{1, i}, \cdots, \boldsymbol{X}_{L-1, i}\right]^{T}$. Through the Map Matrix conversion, $\boldsymbol{x}^{(i)}$ is transformed to $\tilde{\boldsymbol{x}}^{(i)}$.

As shown in Fig. 1, through the Spectrum Sense module, the system can have a well knowledge of the channel state, especially for the interference spectrum distribution. When the threshold is chosen, the Map Matrix $\boldsymbol{P}$ is also decided. The Threshold Decision module adopts the classical single-threshold rule. That is, $P_{n, l}$ is given by

$$
P_{n, l}= \begin{cases}1 & \lambda_{n}<\mathrm{H}, \text { and } x_{l} \text { is mapped to } \tilde{x}_{n} \\ 0 & \text { others }\end{cases}
$$

where $\lambda_{n}$ is the current sensed interference amplitude of the $n$th frequency, and $H$ is the preset threshold. When $\lambda_{n} \geq \mathrm{H}$, it means that the $n$th frequency interference signal is so strong that it will has a heavily bad effect on the communication BER property. So we make $\tilde{x}_{n}=0$ to zero a set of subcarriers where strong interference exists. We take the OFDM-based structure not only to reduce the NBI, but also to carry out the frequency map function. $\boldsymbol{P}$ is the frequency map matrix, and then a $L$-length vector $\boldsymbol{x}^{(i)}$ can be transformed into a $N$-length vector $\tilde{\boldsymbol{x}}^{(i)}$, which can be described as

$$
\tilde{\boldsymbol{x}}_{N \times 1}^{(i)}=\boldsymbol{P}_{N \times L} \times \boldsymbol{x}_{L \times 1}^{(i)}
$$

Where, $\times$ denotes the matrix multiply.

According to the Eq.(7), $P_{n, l}$ represent the current frequency occupation. For $P_{n, l}=1$, the frequency point $x_{l}^{(i)}$ is mapped to the frequency point $\tilde{x}_{n}^{(i)}$. For $P_{n, m}=0$, the frequency point $\tilde{x}_{n}^{(i)}$ whose amplitude is zero. And traditionally, $N$ is larger than $L$. 


\subsection{PARAMETER Pool}

On overview of the system in Fig. 1, we can get a clear idea that the system should has a dynamic parameter set to adjust the system BER performance according to the channel state. So we set up the Parameter Pool module to consider all the parameters comprehensively. To get a better communication between Tx and Rx, the parameters should be the same for Tx and $\mathrm{Rx}$, as shown in Fig. 1. As for the threshold decision, we take the classical single-threshold method to simplify the system and mainly focus on the order $\alpha$ selecting algorithm. Details about the optimization of the WFRFT order $\alpha$ will be discussed in the following part.

According to the signal processing flow and three main modules in Fig. 1, the receiving signal $\tilde{b}(n)$ can be derived as

$$
\tilde{b}(n)=h(n) * b(n)+i(n)+w(n)
$$

Where $h(n)$ is the channel coefficient, $i(n)$ stands for the NBI, and $w(n)$ is the AWGN. And * denotes linear convolution operation. The vector expression of $\tilde{b}(n), \boldsymbol{b}$ is given by

$$
\boldsymbol{b}=\boldsymbol{T}_{\text {acp }} \times \boldsymbol{F}^{-1} \times \tilde{\boldsymbol{X}}
$$

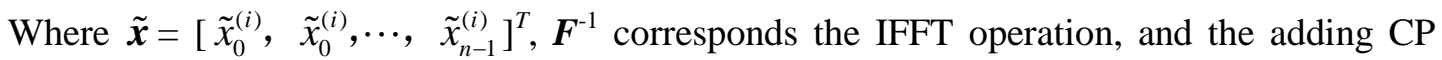
matrix $\boldsymbol{T}_{\text {acp }}$ is

$$
\left.\boldsymbol{T}_{\text {acp }}=\left[\begin{array}{ccccc}
0 & \cdots & 1 & \cdots & 0 \\
\cdots & \cdots & \cdots & \cdots & \cdots \\
0 & 0 & 0 & \cdots & 1 \\
1 & 0 & 0 & \cdots & 0 \\
0 & 1 & 0 & \cdots & 0 \\
0 & 0 & 1 & \cdots & 0 \\
\cdots & \cdots & \cdots & \cdots & \cdots \\
0 & 0 & 0 & \cdots & 1
\end{array}\right]\right\} \mathrm{N}_{\mathrm{cp}}
$$

In the receiving terminal, before demodulate the receiving signal, $\mathrm{CP}$ should be removed, and the removing CP matrix $\boldsymbol{T}_{\text {rсp }}$ is

$$
\boldsymbol{T}_{\text {rcp }}=\left[\begin{array}{cccccccc}
0 & \cdots & 0 & 1 & 0 & 0 & \cdots & 0 \\
0 & \cdots & 0 & 0 & 1 & 0 & \cdots & 0 \\
0 & \cdots & 0 & 0 & 0 & 1 & \cdots & 0 \\
\cdots & \cdots & \cdots & \cdots & \cdots & \cdots & \cdots & \cdots \\
0 & 0 & \underbrace{0}_{\mathrm{N}_{\mathrm{cp}}} & 0 & 0 & \cdots & 1
\end{array}\right]
$$


While for the convenience of analyzing, we assume that the communication system is only influenced by NBI and AWGN, then we can set $h(n)$ to 1 identically. Then, as for the Rx, after removing CP, the receiving signal is demodulated by OFDM and WFRFT module, then $\tilde{\boldsymbol{b}}$ is transformed into $\tilde{\boldsymbol{r}}$, which is given by

$$
\tilde{\boldsymbol{r}}=\boldsymbol{P}^{T} \times \boldsymbol{F} \times \boldsymbol{T}_{\text {rсp }} \times \tilde{\boldsymbol{b}}
$$

Finally, through Despreader and P/S modules, the data demodulation can be fulfilled, and the signal $\boldsymbol{r}$ is output.

\section{Optimization of WFRFT Order}

As for the $k$ th frequency point, $J_{k}$ and $\theta_{k}$ stands for interference signal amplitude and pseudo phase respectively. Let $\boldsymbol{i}=\left[i_{0}, i_{1}, \cdots, i_{\mathrm{N}-1}\right]^{T}, i_{k}=J_{k} e^{j \theta_{k}}$. So through the CMDA-WFrDCS Rx module in Fig. 1, taking the frequency mapping matrix into consideration, the final interference signal $\tilde{\boldsymbol{i}}$ can be given by

$$
\tilde{\boldsymbol{i}}=\boldsymbol{Q} \times \mathbf{i}
$$

Where $\boldsymbol{Q}$ is a $N$-by- $N$ matrix, and $\boldsymbol{Q}=\left[\boldsymbol{q}_{0}, \boldsymbol{q}_{2}, \cdots, \boldsymbol{q}_{N-1}\right]^{T} . \boldsymbol{q}_{k}$ is the $k$ th row of $\boldsymbol{Q}$, when the interference signal is too strong in the $k$ th frequency point, the interference signal in this frequency point should be removed. And the expression of $\boldsymbol{q}_{k}$ is given by

$$
\boldsymbol{q}_{k}= \begin{cases}{[0,0, \cdots, 0]} & \lambda_{k}<H \\ {[\underbrace{0, \cdots, 0,1,0, \cdots, 0]}_{k}} & \lambda_{k} \geq H\end{cases}
$$

Similar to generating Map Matrix $\boldsymbol{P}$ in Eq. (7), $\boldsymbol{Q}$ is the map matrix for the interference signal, while $\boldsymbol{P}$ is just for the transmitted signal. And the function of $\boldsymbol{Q}$ is also to remove the frequency points where strong interference exists.

For the total communication system, the analyzing method and processing flow are the same for the ith column of $\boldsymbol{X}$ in Eq.(2), so instead of $\tilde{\boldsymbol{x}}^{(m)}, \tilde{\boldsymbol{x}}$ is used to stand for the WFRFT-modulated signal to simplify the following derivation process.

After substituting the expression of $\boldsymbol{i}$ and $\boldsymbol{Q}$ into Eq.(14), we can get reduced interference signal in frequency domain expression. Define $r_{\tilde{i}, \tilde{x}}$ as the cross correlation function between $\tilde{\boldsymbol{i}}$ and $\tilde{\boldsymbol{x}}$. And $r_{\tilde{\boldsymbol{i}}, \tilde{\boldsymbol{x}}}$ is given by

$$
r_{\tilde{i}, \tilde{x}}=\frac{\sum_{k=0}^{N-1} \tilde{i}(k)[\tilde{x}(k)]^{*}}{\sqrt{\|\tilde{i}(k)\|^{2} \cdot\|\tilde{x}(k)\|^{2}}}
$$


According to [24], for the single-frequency interference, studies have made sure that the system BER performance and $r_{\tilde{i}, \tilde{x}}$ are inversely related; That is, larger $r_{\tilde{\boldsymbol{i}}, \tilde{x}}$ results in worse performance under the same conditions. It is also true for NBI, since NBI can be regarded as a set of many different single frequency interferences [25].

When $\tilde{\boldsymbol{i}}$ is a deterministic signal, $r_{\tilde{\boldsymbol{i}}, \tilde{\boldsymbol{x}}}$ varies with $\tilde{\boldsymbol{x}}$. Because $\tilde{\boldsymbol{x}}$ a function of $\alpha, r_{\tilde{\boldsymbol{i}}, \tilde{\boldsymbol{x}}}$ is also a function of $\alpha$. The $\sqrt{\|\tilde{i}(k)\|^{2} \cdot\|\tilde{x}(k)\|^{2}}$ can be considered as the normalized coefficient. Finally the expression between $r_{\tilde{\boldsymbol{i}}, \tilde{x}}$ and $\alpha$ is given by

$$
\begin{aligned}
& r_{\tilde{i}, \tilde{x}, m}=\beta \sum_{n=0}^{N-1} \sum_{l=0}^{L-1} \tilde{i}(n)\left[W_{-\alpha}(n, l)\right]^{*} D(l, m), \\
& n=0,1,2 \cdots, N-1, l=0,1,2 \cdots, L-1, m=0,1,2 \cdots, M-1
\end{aligned}
$$

Where $\beta=1 / \sqrt{\|\tilde{i}(k)\|^{2} \cdot\|\tilde{x}(k)\|^{2}}$.

To measure the system performance comprehensively, the mean of $r_{\tilde{i}, \tilde{x}, m}$ is calculated, which can be expressed by

$$
\bar{r}_{\tilde{\boldsymbol{i}}, \tilde{x}}=\frac{1}{M} \sum_{m=0}^{m=M-1}\left|r_{\tilde{\boldsymbol{i}}, \tilde{x}, m}\right|
$$

So the best solution to reduce the interference is to minimize the variable $\overline{\tilde{\boldsymbol{i}}}_{\tilde{\mathrm{x}}, \tilde{x}}$. And the system performance optimization can be described as the optimization of WFRFT order $\alpha$. The optimization is showed as follows

$$
\alpha_{\mathrm{opt}}=\arg \min _{\alpha} \bar{r}_{\tilde{\boldsymbol{i}}, \tilde{\boldsymbol{x}}}
$$

In fact, $\alpha$ varies from 0 to 4 in conventional WFRFT structure, for $\alpha=1,3$, the system is working in time domain, just like in the SC-FDMA. For $\alpha=0$, 2, the system is working in frequency domain, just like in the OFDM structure. According to the symmetry property, the WFRFT matrix in Eq.(3) has similar characteristics in the case where $\alpha$ is in the range [0,1] and the cases where $\alpha$ is in the range [1, 2], [2, 3], or [3, 4]. Moreover, we choose $\alpha$ in the range $[0,1]$ to make the communication system compatible with the current structure, so we conduct the simulation where $\alpha$ is in the range $[0,1]$. When the precision of $\alpha$ is set to $\Delta \alpha$, in order to get the best BER performance, the exhaustive searching method is adopted to select the optimal order, $\alpha_{\mathrm{opt}}$ under the condition that the precision of $\alpha$ is $\Delta \alpha$. 


\section{Experimental Results and Analysis}

In this section, we simulated the CMDA-WFrDCS communication system and verified the optimization performance of the WFRFT order $\alpha$ designed in Section 3. According to the main focus of the paper, we assume that the signal is only influenced by NBI and AWGN, so the channel is generated based on complex Gaussian random process, and $h(n)$ in Eq. (9) is identically equal to 1 . To mitigate most of the Inter-carrier interference (ICI) caused by NBI, the threshold is set to $3 \mathrm{~dB}$. For the channel adopted, we remove The CP and FDE modules to simplify the system and highlight the designed structure and optimization process. The pseudo sequence $\boldsymbol{d}$ is generated by a m-sequence, with length $M=31$. The length of FFT is $N=64$. To reduce the complexity and save the searching time, we also restrict the WFRFT order's searching region to $[0,1]$, with the searching step 0.1 . In order to simulate the communication circumstance with NBI more truly, the relative center frequency of NBI is generated by a random sequence obeying a uniformly distribution in $(0, N)$. The phase of NBI is distributed uniformly in $[0,2 \pi]$. The QPSK baseband modulation is adopted in the system to test the final BER performance.

In order to analyze the effect of WFRFT modulation on NBI more clearly, we give out three interference distribution diagrams in different conditions, which are shown in Fig. 3 , Fig. 4 and Fig. 5 with subchannel samples on the horizontal axis and interference energy on the vertical one. As shown in the Fig. 3, in frequency domain, the original NBI has a severely centralized energy property, which explains the reason why we should take measures to suppress the main interference.

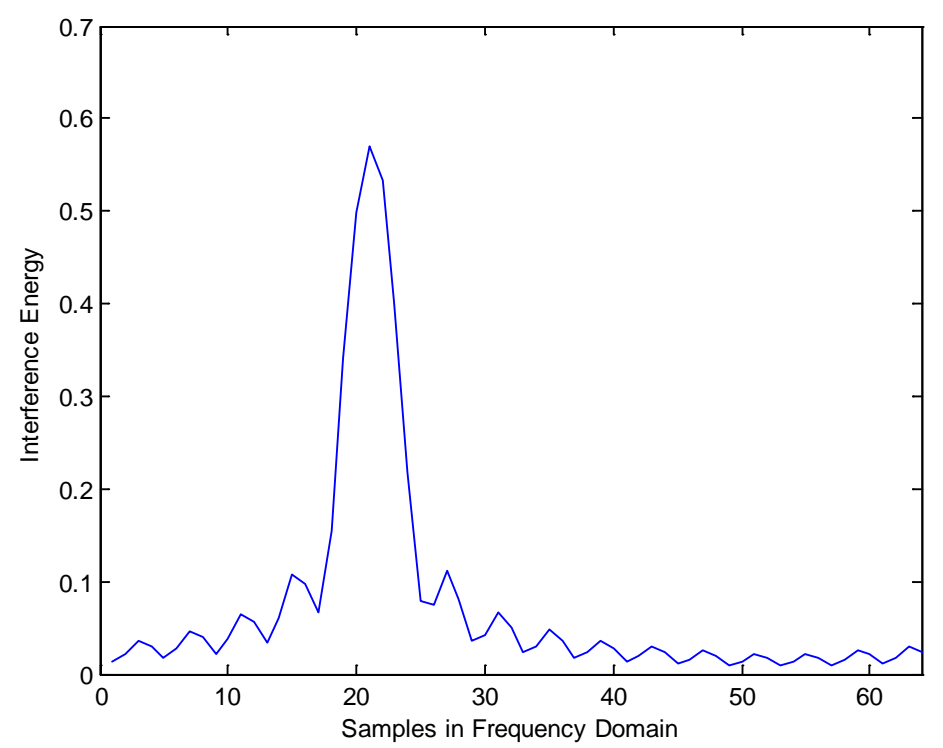

Fig. 3. Original interference energy distribution in frequency domain

Fig. 4 describes the residual interference energy distribution in frequency domain, which is corresponding to Eq.(14). As is shown clearly in Fig. 4, the majority interference energy has been removed, while a few residual interference still exists in other frequency points. And as shown in Fig. 4, the residual interference energy is still unevenly distributed. 
Since the WFRFT is a unitary transformation, the total residual interference energy remains the same for different WFRFT order $\alpha$. However the distribution for it will varies with different WFRFT order $\alpha$. To test the effect of WFRFT order $\alpha$ on the energy distribution, we set $\alpha$ to be $0.1,0.4$ and 0.7 to get the corresponding energy distributions in the WFRFT domain respectively. As is depicted in Fig. 5, we can get a clear idea that the energy distributions vary with different WFRFT order $\alpha$. As is known to all of us, different interference distributions will lead to different BER performance. So we have good reasons to believe that there must be a certain WFRFT order $\alpha$ to make the residual interference energy distributed more uniform, which means that the effect of residual interference can be suppressed at the greatest extent.

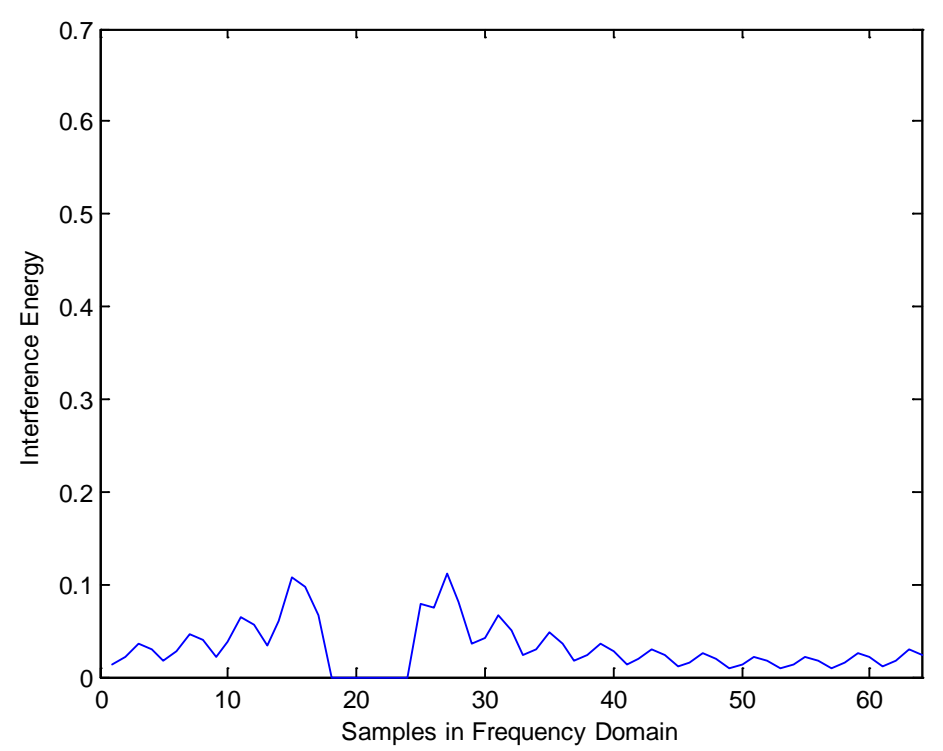

Fig. 4. Residual interference energy distribution in frequency domain

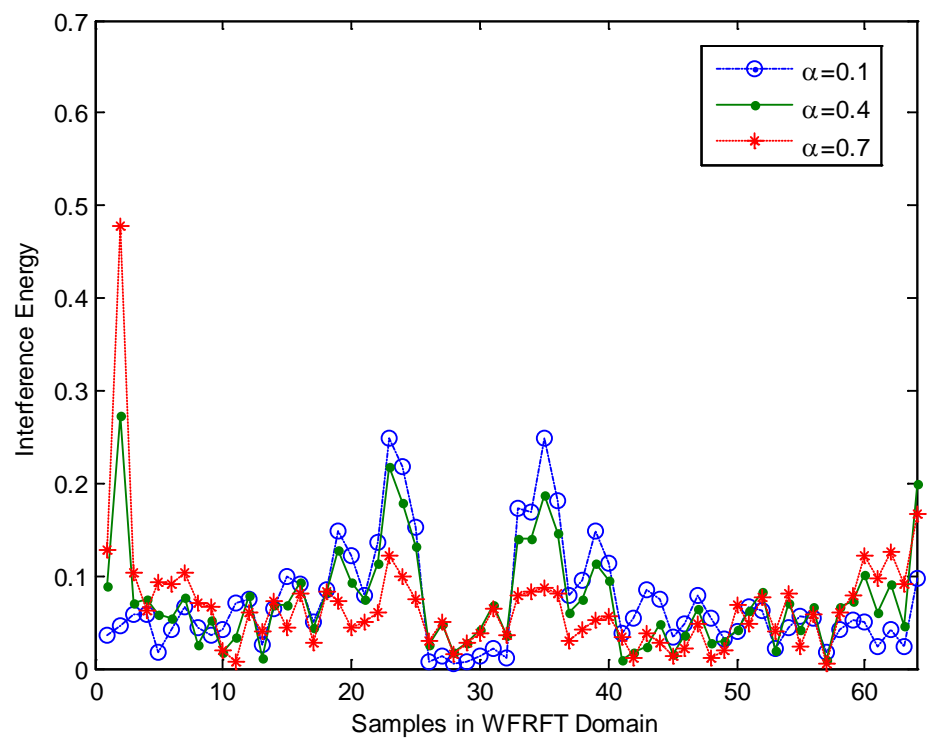

Fig. 5. Residual interference energy distribution in WFRFT domain 
To test the BER performance of different $\alpha$ selections against signal-to-noise ratio (SNR), the jam-to-signal ratio (JSR) is set to $7 \mathrm{~dB}$, and SNR varies from -1 to $6 \mathrm{~dB}$. Exhaustive searching method is used to find the optimal $\alpha$ in the designed structure. As depicted in Fig. 6, three typical values of $\alpha$ are chosen to test the system performance. And the best BER performance can be achieved when $\alpha$ is equal to 0.4. In Fig. 6, the blue dashed line with a square, the green solid line with a pentagram and the red dotted line with a circle represent the BER performance for $\alpha$ are equal to 0, 0.4 and 1 respectively. At SNR $<0 \mathrm{~dB}$, for $\alpha=0,0.4$, the BER performances are very close, while they both outperform the BER performance with $\alpha=1$. This is because that AWGN is strong enough to cause the bit error heavily. In consideration of the NBI influence, the dispersion degree in frequency domain has a significant effect on the BER performance. The more disperse, the worse the BER performance. At SNR $>1.5 \mathrm{~dB}$, for $\alpha=0,1$, the BER performance curves will run into the error floors, with BER almost equaling to $3.5^{*} 10^{-3}$ and $5.4 * 10^{-3}$ respectively. While for $\alpha=0.4$, the BER performance curve continues declining till SNR $>4 \mathrm{~dB}$. The error floors could be attributed to the influence of NBI. For $\alpha=0.4$, the error floor will comes later because the influence of NBI can be reduced to the lowest.

Besides, to compare the performance of the CDMA-WFrDCS system and the one in [10], we also give out the BER performance of the system in [10]. As shown in Fig. 6, the black dash-dot line with a cross represents the BER performance of the system in [10] under the same constraints. The CMDA-WFrDCS system achieves better performance than the one in [10], this is mainly because the PN sequence helps to spread the interference more widely.

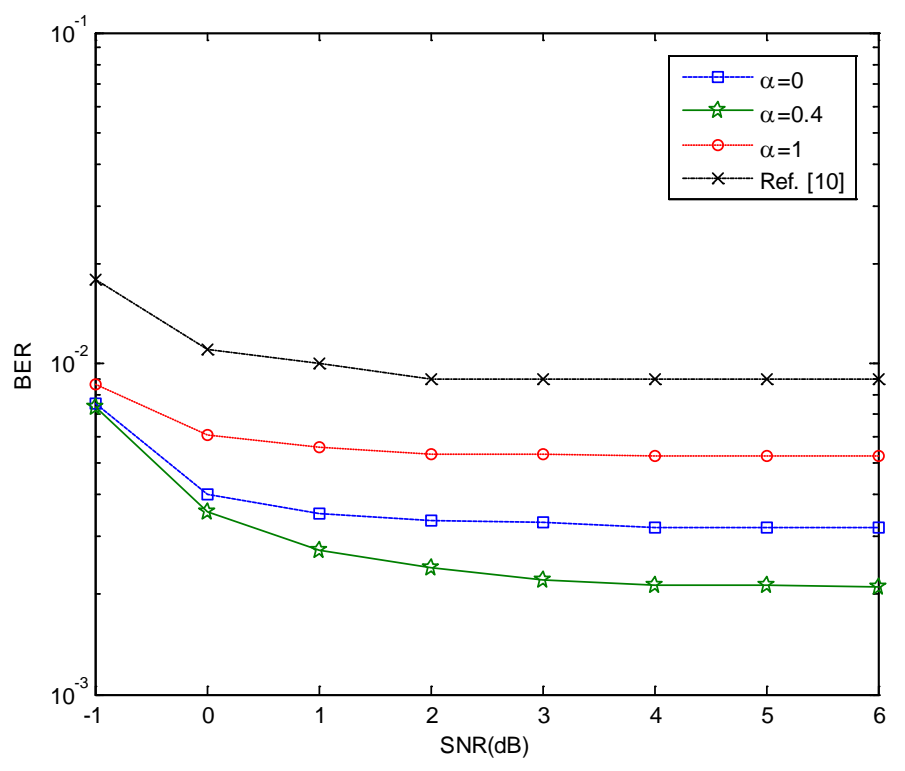

Fig. 6. BER performance of different $\alpha$ selections against signal-to-noise ratio

To test the BER performance of different jam-to-signal ratio (JSR) against different $\alpha$ selections, SNR is set to 9dB. When JSR is set to different values, Fig. 7 depicts the BER performance with the WFRFT order $\alpha$, which varies from 0 to 1 with a searching step 0.1 . As shown in Fig. 7, the system exhibits different $\alpha$-optimizing trends when the JSR is different. At JSR $>12$, when the NBI energy is strong, the original signal is more easily contaminated. The optimal $\alpha$ is chosen to make the system work in frequency domain, which is closer to 1 . 
At JSR $<12$, when the NBI energy is weak, the original signal is contaminated little by NBI. So the optimal $\alpha$ is realized close to time domain, which is closer to 0 . Besides, stronger interference doesn't necessarily mean worse BER performance. As depicted in Fig. 7, the red solid line with a square and the black dotted line with a circle represent the communication channels with JSR=10, 11 respectively. The total system BER performance of curve at $\mathrm{JSR}=11$ is better than that of curve at JSR $=10$, even though the former JSR is larger. This is mainly caused by the number of available subcarriers, $L$ in Eq.(7). Compared with the system for JSR $=10$, It's true that the NBI energy is stronger for JSR=11. However, $L$ is set to be 58 and 59 for JSR=11, 10 respectively. So the total residual NBI energy for JSR=11 is weaker than that of the system for JSR $=10$.

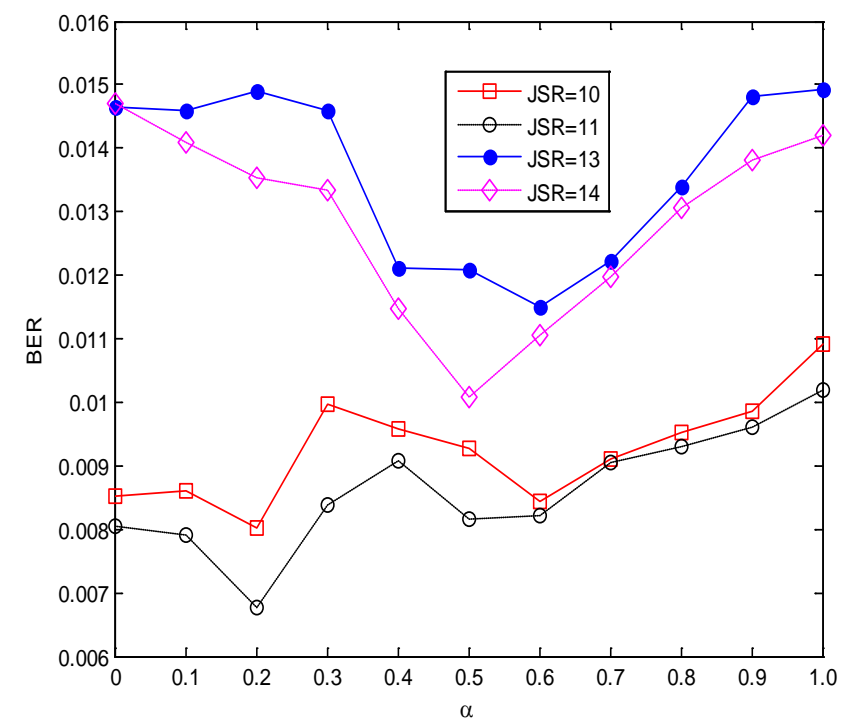

Fig. 7. BER performance of different jam-to-signal ratio against different $\alpha$ selections

To further verify the designed system's performance, we also conduct the simulations to get the BER performances of other baseband modulations, such as BPSK, 16QAM and 64QAM. Except for the baseband modulations, the simulation conditions are the same with Fig. 6, that is $\mathrm{JSR}=7 \mathrm{~dB}, \mathrm{SNR}=[-1,6] \mathrm{dB}, \alpha_{\mathrm{opt}}=0.4$. As illustrated in Fig. 8, with the increase of the baseband modulation order $K$, the BER performance will get worse correspondingly. This can be attributed to the distance reduction of the constellation neighboring points for different baseband modulations. As shown in Fig. 1 and Eq.(19), we build our optimization program and solve it on the premise that the NBI has been largely eliminated and the residual interference remains small enough, so the BER performances are poor for 16QAM and 64QAM in Fig. 8. And the effective solution is to increase the original transmitting signal's power or decrease the baseband modulation's order $K$, which also shows that there is a compromise between system's effectiveness and reliability. 


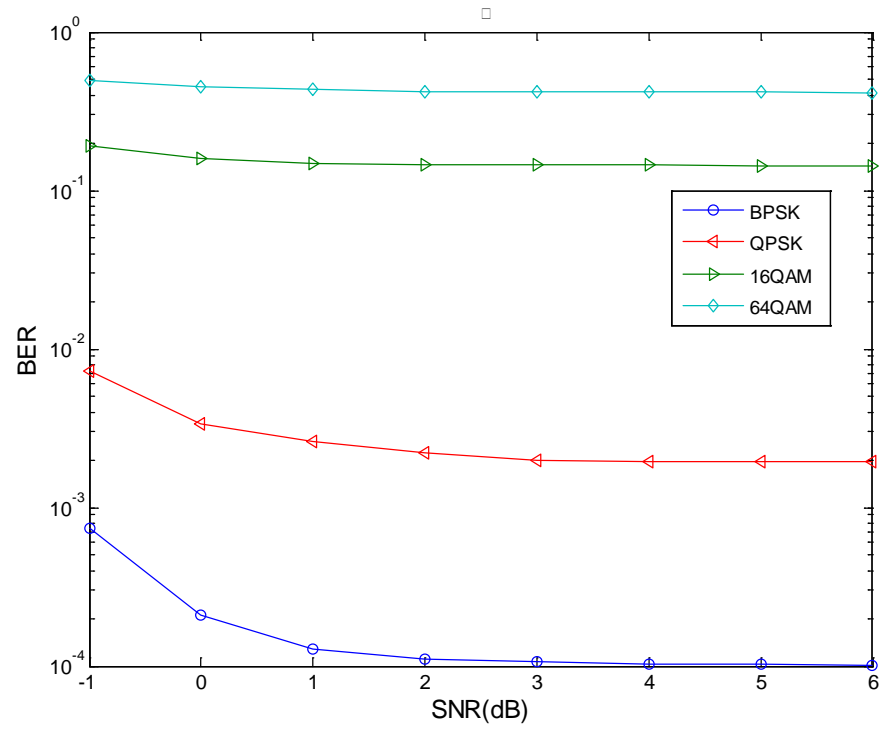

Fig. 8. BER performance of different baseband modulations

\section{Conclusion and Future Work}

In this paper, we propose a new scheme to suppress the NBI utilizing CDMA and WFRFT domain preprocessing technologies in OFDM-based systems. Then we exhibit the total CMDA-WFrDCS structure and its three main submodules in details. We also deduce the WFRFT optimal order's expression to eliminate the residual NBI to the maximum extent. Simulation results show that the proposed scheme can achieve a better BER performance than SC or MC with CDMA and frequency domain preprocessing in terms of the narrowband interference suppression.

In our future work, smart sensing threshold will be adopted to make a tradeoff between the BER performance and the frequency efficiency. Other practical channels such as flat Rayleigh and multipath Rayleigh fading channels will also be implemented to more fully test the BER performance of the proposed scheme.

\section{References}

[1] S. Sesia, I. Toufik, and M. Baker, LTE-the UMTS long term evolution, John Wiley, 2015.

[2] B. Furht, and S. A. Ahson, Long Term Evolution:3GPP LTE radio and cellular technology, Crc Press, 2016.

[3] I. Kashiwamura, S. Tomita, K.Komatsu, N. H. Tran, H. Oguma, N. Izuka, S. Kameda, T. Takagi, and K. Tsubouchi, "Investigation on single-carrier and multi-carrier hybrid system for uplink," in Proc. of IEEE 20th International Symposium on Personal, Indoor and Mobile Radio Communications, pp. 3188-3192, September, 2008. Article (CrossRefLink) .

[4] L. Mei, X. Sha, and N. Zhang, "The approach to carrier scheme convergence based on 4-weighted fractional fourier transform,” IEEE Communications Letters, vol. 14, no. 6, pp. 503-505, 2010. Article (CrossRefLink) .

[5] L. Mei, X. Sha, Q. Zhang and N. Zhang, "The concepts of hybrid-carrier scheme communication system," in Proc. of Communications and Networking in China (CHINACOM), 2011 6th International ICST Conference on, pp. 26-33, August, 2011. Article (CrossRefLink) . 
[6] Y. Hui, B. Li, and T. Zhao, "4-weighted fractional Fourier transform over doubly selective channels and optimal order selecting algorithm," Electronics Letters, vol. 51, no. 2, pp. 177-179, 2015. Article (CrossRefLink) .

[7] X. Sha, X. Qiu, and L. Mei, "Hybrid carrier CDMA communication system based on weighted-type fractional Fourier transform," IEEE Communications Letters, vol. 16, no. 4, pp. 432-435, 2012. Article (CrossRefLink).

[8] X. Qiu, X. Sha, and L. Mei, "Performance of hybrid carrier DS CDMA communication system," Communications and Networking in China (CHINACOM), 2011 6th International ICST Conference on , pp. 176-180, 2011. Article (CrossRefLink) .

[9] Z. Wang, L. Mei, X. Wang, X. Sha and N. Zhang, "BER analysis of hybrid carrier system based on WFRFT with carrier frequency offset," Electronics Letters, vol. 51, no. 21, pp. 1708-1709, 2015. Article (CrossRefLink) .

[10] L. Mei, Q. Zhang, X. Sha and N. Zhang, "WFRFT Precoding for Narrowband Interference Suppression in DFT-Based Block Transmission Systems," IEEE Communications Letters, vol. 17, no. 10, pp. 1916-1919, 2013. Article (CrossRefLink) .

[11] S. Yu, H. Dai, K. Wu, X. Cheng and C. Xu, "Performance Analysis for WFRFT-OFDM Systems to Carrier Frequency Offset in Doubly Selective Fading Channels," in Proc. of Intelligent Control and Information Processing(ICICIP), 2015 Sixth International Conference on, pp. 6-10, 2015. Article (CrossRefLink) .

[12] S. B. Weinstein, "The history of orthogonal frequency-division multiplexing [History of Communications]," IEEE Communications Magazine, vol. 47, no. 11, pp. 26-35, 2009. Article (CrossRefLink) .

[13] M. Morelli, and M. Moretti, "Improved decoding of BICM-OFDM transmissions plagued by narrowband interference," IEEE transactions on wireless communications, vol. 10, no. 1, pp. 20-26, 2011. Article (CrossRefLink) .

[14] D. Zhang, P. Fan, and Z. Cao, "Interference cancellation for OFDM systems in presence of overlapped narrow band transmission system," IEEE Transactions on Consumer Electronics, vol. 50, no. 1, pp. 108-114, 2004. Article (CrossRefLink) .

[15] D. Darsena, and F. Verde, "Successive NBI cancellation using soft decisions for OFDM systems," IEEE Signal Processing Letters, pp. 873-876, 2008. Article (CrossRefLink) .

[16] A. J. Coulson, "Bit error rate performance of OFDM in narrowband interference with excision filtering," IEEE transactions on wireless communications, vol. 5, no. 9, pp. 2484-2492, 2006. Article (CrossRefLink).

[17] J. Zhang, and J. Meng, "Robust narrowband interference rejection for power-line communication systems Using IS-OFDM,” IEEE Transactions on Power Delivery, vol. 25, no. 2, pp. 680-692, 2010. Article (CrossRefLink) .

[18] J. Zhang, and J. Meng, "Noise resistant OFDM for power-line communication systems," IEEE transactions on Power Delivery, vol. 25, no. 2, pp. 693-701, 2010. Article (CrossRefLink) .

[19] D. Gerakoulis, and P. Salmi, "An interference suppression OFDM system for ultra wide bandwidth radio channels," Ultra bandwidth systems and technologies, IEEE Conference on, pp. 259-264, 2002. Article (CrossRefLink) .

[20] D. Gerakoulis, and P. Salmi, "An interference suppressing OFDM system for wireless communications," in Proc. of Communications, 2002. ICC 2002. IEEE International Conference on, vol. 1, pp. 480-484, 2002. Article (CrossRefLink) .

[21] Z. Wu, and C. R. Nassar, "Narrowband interference rejection in OFDM via carrier interferometry spreading codes," IEEE Transactions on Wireless Communications, vol. 4, no. 4, pp. 1491-1505, 2005. Article (CrossRefLink) .

[22] C. C. Shih, "Fractionalization of Fourier transform," Optics communications, vol. 118, no. 5, pp. 495-498, 1995. Article (CrossRefLink).

[23] Q Ran, D. S. Yeung, E. C. C. Tsang and Q. Wang, "General multifractional Fourier transform method based on the generalized permutation matrix group," IEEE Transactions on Signal Processing, vol. 53, no. 1, pp. 83-98, 2005. Article (CrossRefLink) .

[24] X. Qiu, X. Sha and L. Mei, "Hybrid Carrier Spread Spectrum System Based on 4-Weighted Fractional Fourier Transform,” China Communications, vol. 9, no. 1, pp. 13-19, 2012. 
[25] T. Zhao, H. Zhao, Y. Zhao, X. Cheng and B. Ai, “A frequency-domain estimation scheme for single-tone interference in OFDM systems,” in Proc. of 2011 Third International Conference on Communications and Mobile Computing, 2011. Article (CrossRefLink) .

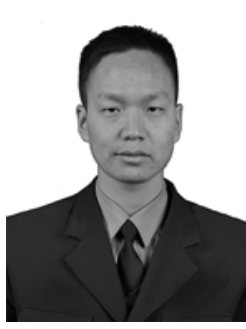

Yuan Liang received the B.Sc. degree in information and navigation engineering and the M.A.Sc. degree in information and communication engineering from Air Force Engineering University, China, in 2012, and 2015, respectively. He is currently pursuing his Ph.D. at Institute of Information and Navigation, Air Force Engineering University, Xi'an, China. His research interests include intelligent signal processing, wireless communications, secure communications, optimization and intelligent algorithm.

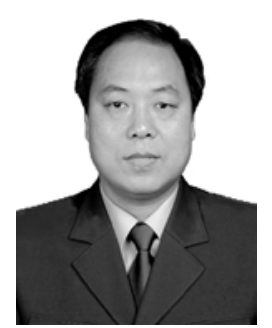

Xinyu Da received the B.Sc. in Xidian University, the M.A.Sc. degree in communication and electronic system from Air and Missile Defense College, and the Ph.D. degree from School of Marine Science and Technology, NPU in 1983, 1988 and 2007 respectively. He is working as the professor in Information and Navigation College, AFEU. His research interests include Satellite communications, communication theory, signal processing, transform domain communication system and cognitive radio.

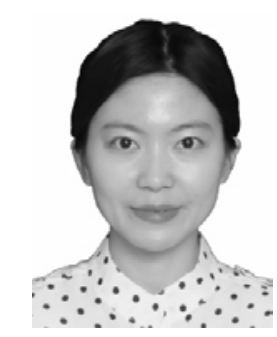

Shu Wang was born in 1987. She received B.Sc. degree in Xidian University in 2010, her M. A.Sc. degrees and Ph.D. degree in circuits and system from Air Force Engineering University in 2013 and 2016 respectively. Her research interests are Physical layer security and Communication Signal Processing. 